\title{
Relação Fraternal e Síndrome de Down: um Estudo Comparativo
}

\author{
Nara Liana Pereira-Silva \\ Universidade Federal de Juiz de Fora, MG, Brasil. \\ Bruna Rocha de Almeida \\ Universidade Federal de Juiz de Fora, MG, Brasil.
}

\author{
Sarah de Rezende Crolman \\ Universidade Federal de Juiz de Fora, MG, Brasil. \\ Mayse Itagiba Rooke \\ Universidade Federal de Juiz de Fora, MG, Brasil.
}

Resumo: As relações fraternais em famílias com filhos com e sem síndrome de Down (SD) têm sido pouco investigadas no Brasil. Este estudo descreve o funcionamento familiar e compara a relação entre irmãos em dois tipos de famílias, identificando similaridades e diferenças nos relatos dos genitores. Participaram do estudo oito famílias compostas por mãe, pai e dois filhos, sendo que em quatro delas havia somente membros com desenvolvimento típico (DT) e nas demais havia um membro com SD, totalizando oito díades de irmãos, de ambos os sexos com idades entre cinco a 15 anos. Utilizou-se o Questionário de Caracterização do Sistema Familiar, que foi respondido pelas mães, e entrevistas semiestruturadas, cujos genitores responderam. Os resultados indicam similaridades na rotina diária das famílias, sendo as mães as principais responsáveis pelas tarefas domésticas e cuidados com os filhos, em ambos os tipos de famílias. As relações fraternais foram descritas, pelos pais e mães, como Mistas e Amistosas em ambos os tipos de famílias, caracterizando, portanto, como sendo positivas. O filho com DT foi indicado como líder na maioria das famílias com um membro com SD, enquanto nas famílias com as díades com DT, a maioria dos genitores indicou o filho mais velho. Os dados convergem com os da literatura acerca do fato de que a relação entre a díade de irmãos SD-DT tende a ser positiva, com características de amistosidade mais do que conflitos. Os dados encontrados sugerem coerência com os da literatura atual.

Palavras-chave: Relação Fraternal, Síndrome de Down, Irmãos.

\section{Sibling Relationship and Down Syndrome: A Comparative Study}

\begin{abstract}
The sibling relationships in families with children with and without Down syndrome (DS) have been poorly investigated in Brazil. This study describes family functioning and compares the relationship between siblings in two types of families, identifying similarities and differences in the reports of the parents. Eight families $(n=8)$ consisting of mother, father and two children participated on the study. In four of them, there were only typically developing (TD) members and in the others there was a member with DS, with a total amount of eight dyads of siblings of both sexes, ranging from five to 15 years old. The Family System Characteristics Questionnaire was answered by the mothers and semi-structured interviews were responded by the parents. The results indicated similarities in the daily routine of the families, in which mothers are primarily responsible for household chores and child care in both types of families. Sibling relations were described by fathers and mothers as Mixed and Friendly in both types of families, therefore, characterizing them as positive. The child with TD was indicated as a leader in most families with a member with DS, and in families with TD dyads, most parents indicated their eldest son. Data converge with those in the literature about the fact that the relationship between the dyad of DS-TD siblings tends to be positive with characteristics of friendship rather than conflict.
\end{abstract}

Keywords: Sibling Relationship, Down syndrome, Siblings. 


\title{
Relación Fraternal y Síndrome de Down: Un Estudio Comparativo
}

\begin{abstract}
Resumen: Las relaciones fraternales en familias con hijos con y sin síndrome de Down (SD) han sido poco investigadas en Brasil. Este estudio describe el funcionamiento familiar y compara la relación entre hermanos en dos tipos de familias, uno compuesto por miembros con y sin SD y el otro con todos los miembros con desarrollo típico (DT), identificando semejanzas y diferencias en los relatos de padres y madres en estos tipos de familias. Participaron del estudio ocho familias compuestas por madre, padre y dos hijos. En cuatro de estas solamente había personas con desarrollo típico (DT) mientras que en las otras había una persona con síndrome de Down, con un total de ocho diadas de hermanos de ambos sexos, con edades entre 5 a 15 años. Se utilizaron el Cuestionario de Caracterización del Sistema Familiar, que fue respondido por las madres; y entrevistas semiestructuradas, respondidas por los progenitores. Los resultados indican semejanzas en la rutina diaria de las familias, siendo las madres las principales responsables por las tareas domésticas y cuidados con los hijos en los dos tipos de familias. Las relaciones fraternales fueron descritas, por padres y madres, como complementarias y de amistad, siendo caracterizadas, por lo tanto, como positivas. El hijo con DT fue caracterizado como líder en la mayoría de las familias con una persona con SD, y en las familias con las diadas con DT, la mayoría de los progenitores caracterizaron al hijo mayor. Los datos se muestran coherentes con los de la literatura sobre el hecho de que la relación entre las diadas de hermanos SD-DT tiende a ser positiva con amistad y con poco conflicto.
\end{abstract}

Palabras clave: Relación Fraternal, Síndrome de Down, Hermanos.

\section{Introdução}

O nascimento de uma criança com síndrome de Down (SD) traz implicações ao funcionamento familiar. Nesse sentido, a literatura (Dodd, Zabriskie, Widmer, \& Eggett, 2009; Lemes, \& Barbosa, 2007; Messa, \& Fiamenghi Jr., 2010; Nunes, \& Dupas, 2011) salienta que os genitores, em geral, apresentam dúvida e medo sobre os cuidados e o futuro do filho. Em algumas famílias pode ocorrer a aproximação entre seus membros (Voivodic, \& Storer, 2002), entretanto, mesmo ocorrendo a coesão do grupo, ela se torna difícil, porque a criança requer cuidados e exige muita disponibilidade da pessoa que cuida dela (Casarin, 1999). Vale ressaltar que a adaptação ao novo membro ocorre de forma particular para cada família, dependendo dos recursos psicológicos de cada membro, bem como do grupo. É comum que os genitores questionem suas capacidades de tomar decisões, duvidando de suas potencialidades para cuidar do bebê, podendo assim, comprometer a relação de cuidado parental e a formação do vínculo, o que pode causar impactos negativos no bem-estar da família (Dodd et al., 2009) e, portanto, nas relações familiares (Burke, 2010).
Embora alguns estudos tenham se dedicado às investigações sobre as relações estabelecidas no contexto familiar, a maior parte das pesquisas sobre família focaliza exclusivamente a mãe como participante (Bailey Jr., 2007; Grisante, \& Aiello, 2012) e avalia prioritariamente a interação mãe-filho com SD. Stoneman (2009) ressalta que tem sido recente o interesse de pesquisadores pelo subsistema fraternal em famílias com membros com deficiência intelectual (DI) e, evidentemente, naquelas com membros com SD, já que a DI é uma das principais características dessas pessoas. Ainda que se reconheça um aumento de estudos sobre esse subsistema familiar (Aguayo, 2010; Cuskelly, 2016; Iriarte, \& Ibarrola-García, 2010; Mandleco, \&Webb, 2015; Rossetti, \& Hall, 2015; Stoneman, 2005, 2009; Tomeny, Ellis, Rankin, \& Barry, 2017), há questões destacadas por Stoneman (2005) que mantêm esse campo de investigação como uma área emergente, como por exemplo, a necessidade de estabelecer um padrão de relacionamento entre irmãos quando um deles tem DI. Nessa questão, Stoneman salienta a importância dos estudos comparativos, atentando-se para o rigor metodológico necessário na condução dos mesmos. Dada à natureza sistêmica do funcionamento familiar, destaca-se a relevância 
de investigar os irmãos, assim como o pai, a mãe e os demais membros que compõem o grupo, considerando a diversidade de famílias existentes na atualidade para melhor compreensão das características e inter-relações existentes nesse grupo. Ressalta-se, no entanto, que o presente estudo focaliza o subsistema fraternal. Nesse sentido, investigar esse subsistema em famílias com membros com SD se mostra como prioridade na agenda de pesquisa em desenvolvimento familiar, especialmente, no Brasil.

Stoneman (2009) destaca a importância da utilização de uma multiplicidade metodológica ao planejar pesquisas com o objetivo de comparar irmãos de crianças com deficiência e irmãos de crianças com desenvolvimento típico (DT), uma vez que existem peculiaridades entre os grupos e que nem sempre os pesquisadores estão atentos a elas. Nesse sentido, para que se obtenham resultados satisfatórios ao realizar pesquisas comparativas com irmãos, é preciso atentar para diferentes variáveis, tais como ordem de nascimento e diferença de idade entre os irmãos, sexo dos participantes, etiologia e grau de severidade da DI, renda familiar, estrutura familiar, dentre outras (Meyers, \& Vipond, 2005; Stoneman, 2005; 2009). O controle dessas variáveis permite uma comparação entre grupos mais eficaz e possibilita a produção de resultados confiáveis.

No tocante aos resultados dos estudos sobre irmãos, estes são consistentes ao fato do irmão com DT assumir o papel de irmão mais velho quando na presença do irmão com DI ou com SD, independentemente da idade e da ordem de nascimento do irmão com DT (por exemplo: Abramovitch, Stanhope, Pepler, \& Corter, 1987; Hannah, \& Midlarsky, 2005; Núnez, \& Rodríguez, 2005; Shivers, \& Dykens, 2017; Stoneman, Brody, Davis, Crapps, \& Malone, 1991). Aliado a esse aspecto, é comum que os papéis exercidos pelos irmãos sejam assimétricos, sendo que o irmão com DT tende a assumir comportamentos diretivos e de supervisão em relação a seu irmão com SD (Pereira-Silva, 2003). Além disso, há evidências de que os irmãos com DT se envolvem, direta e indiretamente, nas atividades de cuidado do irmão com DI, não havendo dados de que eles sejam significativamente prejudicados em suas atividades sociais, lúdicas e escolares (Floyd, Costigan, \& Richardson, 2016, Nunes, 2006; Povee, Roberts, Bourke, \& Leonard, 2012; Shivers, \& Dykens, 2017; Stoneman et al., 1991). Os resultados de Cuskelly e Gunn (2003) e de McHale e Gamble (1989) mostram maior envolvimento dos irmãos com DT nas atividades de cuidado com o irmão com DI do que no grupo de ambos os irmãos com DT, segundo os relatos dos genitores. Ademais, a literatura aponta dados controversos em relação às diferenças de gênero e o desempenho das atividades de cuidado. Nesse sentido, há estudos mostrando que as meninas realizam mais atividades domésticas e apresentam maior frequência de comportamentos de cuidado e ajuda do que os meninos na presença de um irmão com deficiência (Gomes, \& Bosa, 2004; Hannah, \& Midlarsky, 2005; Petean, \& Suguihura, 2005; Soares, Franco, \& Carvalho, 2009). Todavia, há investigações anteriores que não encontraram evidências que comprovassem essa diferença (Abramovitch et al., 1987; Brody, Stoneman, Davis, \& Crapps, 1991).

Os dados de Choi e Van Riper (2014) com mães coreanas mostram que a adaptação do irmão com DT nas famílias com filho com SD é tão boa quanto a adaptação em famílias apenas com filhos com DT, considerando diferentes áreas, tais como, consciência, sentimento, divertimento, ajuda e defesa. Entretanto, comparando esses dados com estudos similares com mães coreanas que moram nos Estados Unidos da América (EUA), verifica-se que as mães na Coreia percebem a relação fraternal como menos desejável ou menos saudável (menos ideal) do que as mães caucasianas nos EUA, sugerindo que os irmãos com DT na Coreia experienciam mais problemas nas áreas mencionadas do que aqueles que moram nos EUA. Esse resultado reforça a necessidade de mais estudos acerca das relações fraternais em tais famílias e, também, atenta para importância da condução de estudos transculturais.

No que se refere à qualidade da relação fraternal, os dados mostram que os irmãos com DT são como afetuosos e tolerantes nas interações, sendo menos competitivos e apresentando menos comportamentos agressivos quando comparados às díades compostas por ambos os irmãos com DT (Soares et al., 2009). Além disso, a relação entre a díade de irmãos com DI e com DT tende a ser positiva, com características de amistosidade e sincronia (Abramovitch et al., 1987; Orsmond, \& Seltzer, 2007; Pereira-Silva, 2003). Os irmãos com DT tendem a apresentar maior frequência de comportamentos pró-sociais direcionados a seus irmãos com SD quando comparados às díades de irmãos com DT (Abramovitch et al., 1987). Devido à interação com o irmão com DI, alguns estudos indicam que os irmãos com DT podem se tornar mais empáticos, altruístas e 
solidários (Gomes, \& Bosa, 2004; Messa, \& Fiamenghi Jr., 2010), adquirindo melhores estratégias de enfrentamento, o que pode ter impacto positivo sobre a sua saúde mental. Por outro lado, há pesquisas que apontam efeitos negativos da convivência com um irmão com DI, tais como depressão, ansiedade, problemas de comportamento (McHale, \& Gamble, 1989) e dificuldade de relacionamento com os pares (Burke, 2010; Inam, \& Zehra, 2012). A qualidade da relação entre irmãos merece mais aprofundamento, uma vez que as pesquisas apontam resultados inconsistentes.

No estudo de Mulroy, Robertson, Aiberti, Leonard e Bower (2008), os genitores relataram vantagens da convivência entre irmãos com SD e com DT, apontando implicações positivas em relação às características de personalidade, habilidades e competências, tais como, maior tolerância e aceitação de pessoas com deficiência, desenvolvimento de maior empatia e maturidade emocional, paciência. Além dessas características, os genitores afirmaram que seus filhos com DT eram notavelmente mais compassivos, atenciosos e gentis. No que se refere ao cuidado com o irmão com SD, os genitores percebiam que os filhos com DT facilmente os auxiliavam nessa atividade e esse tipo de comportamento era generalizado para fora do contexto doméstico. O desenvolvimento de valores essenciais à vida foi relatado como sendo uma vantagem importante para os irmãos com DT e que era estimulado pelos próprios genitores. Mulroy et al. (2008) destacaram como desvantagens dessa convivência, segundo os genitores: prejuízos na socialização dos irmãos, dificuldade em promover atividade de lazer/recreação de forma conjunta com os filhos (com SD e com DT), restrições financeiras, a carga sobre os irmãos com DT no que se refere à assistência com o cuidado do irmão com SD, o fato de os irmãos com DT não conviverem com um irmão com DT, enfrentar os problemas de comportamento dos irmãos com SD e, ainda lidar com mal-entendidos acerca da síndrome e de situações de preconceito.

Estudos mostram ainda que o bem-estar do irmão com DT pode ser muito influenciado pelos seguintes fatores: as exigências e os desafios que estão associados aos cuidados de uma criança com SD (demandas familiares), a percepção da família acerca do filho com SD (avaliação da família), a forma como a família lida com as dificuldades (estratégias familiares de resolução de problemas e de enfrentamento) e os recursos disponíveis à família (Choi, \& Van Riper, 2014). Nesse sentido, há diferentes variáveis/fatores que se inter-relacionam e que têm implicações na dinâmica e funcionamento familiar e, consequentemente, nas relações fraternais, embora as pesquisas ainda mostrem resultados inconsistentes.

O panorama apresentado indica a importância de mais estudos sobre as relações fraternais, considerando a necessidade de aprofundamento do tema no contexto brasileiro. De acordo com a perspectiva sistêmica, a família deve ser pesquisada considerando-a um grupo, com um funcionamento próprio. Dada à relevância das relações fraternais para a dinâmica e funcionamento familiar, de acordo com a perspectiva sistêmica e considerando a importância da participação do pai com filho com SD (Henn, \& Sifuentes, 2012), o presente trabalho tem como objetivo descrever o funcionamento familiar e comparar a relação entre irmãos em dois tipos de famílias, uma composta por membros com e sem SD e a outra com todos os membros com DT, identificando similaridades e diferenças nos relatos dos genitores em ambos os tipos de família.

Destaca-se que a inclusão da mãe e do pai como participantes neste estudo representa uma tentativa de melhor compreender o grupo, o que está em acordo com teorias e modelos mais complexos de análise do desenvolvimento humano e familiar.

\section{Método}

Este tópico descreve os aspectos metodológicos que foram adotados nesta investigação, destacando os participantes, os instrumentos e os procedimentos para coleta e análise de dados. Ressalta-se que se trata de um estudo descritivo e comparativo realizado em ambiente natural.

\section{Participantes}

Participaram deste estudo oito famílias compostas por mãe, pai e uma díade de filhos, sendo quatro díades compostas por um filho com o diagnóstico de SD e seu irmão com DT; e quatro díades compostas por ambos os filhos com DT. Todos os membros da família moravam juntos em uma cidade do interior de Minas Gerais.

As oito díades eram compostas por irmãos do mesmo sexo, sendo quatro díades do sexo masculino ( $\mathrm{SD}-\mathrm{n}=2 ; \mathrm{DT}-\mathrm{n}=2$ ) e quatro do sexo feminino ( $\mathrm{SD}-\mathrm{n}=2$; DT $-\mathrm{n}=2$ ), com idades variando entre cinco a 15 anos. A média da idade dos participantes com SD e do irmão focal nas díades DT era de 8,5 anos e de seus irmãos, em ambos os grupos, a média era de 
10,5 anos de idade, havendo similaridade nas idades das díades de irmãos nos grupos SD e DT. A diferença máxima de idade entre os membros das díades era de cinco anos. Para identificação dos grupos de família foi utilizada a letra F (família) seguida pela sigla SD (síndrome de Down - FSD) para as famílias com um membro com SD e outro com DT; e DT (desenvolvimento típico - FDT) para as famílias com ambos os membros com DT. A letra D foi utilizada para a denominação de Díade e a letra I para designar Irmão, assim, D1 se trata da díade 1 em ambas as famílias, sendo I-SD o irmão com síndrome de Down e I-DT o irmão com desenvolvimento típico em ambas as famílias. A Tabela 1 especifica dados da composição das díades, considerando cada uma nas respectivas famílias.

Nas famílias com membros com SD, a idade média das mães era de 44,5 anos e dos pais, 47 , e, nas famílias com ambos os membros com DT, de 43 e 46 anos, respectivamente. Com exceção de um pai que era aposentado (FDT) e uma mãe (FDT), todos os demais genitores trabalhavam fora. Os pais trabalhavam em média 10,25 horas por dia no grupo FSD e 13 horas diárias no grupo FDT. Em relação às mães, somente uma não trabalhava (FDT). A média diária do tempo que as mães dedicavam ao trabalho era de 6,45 horas por dia nas famílias FSD e 6,33 horas por dia nas outras famílias. A renda familiar nas famílias FSD variou de 1,6 a 23,5, com média de 8,1 salários mínimos, enquanto nas famílias FDT variou de 2,6 a 20, com média de 8,3 salários mínimos.

\section{Instrumentos}

Foram utilizados os seguintes instrumentos: Questionário de Caracterização do Sistema Familiar (Dessen, 2009) e entrevista semiestruturada. O Questionário investigou dados demográficos, incluindo informações sobre escolaridade e ocupação dos genitores, renda familiar, bem como dados relativos à caracterização do sistema familiar, atividades de lazer e de rotina da família. Já a entrevista teve como objetivo investigar a percepção dos genitores acerca da relação fraternal estabelecida entre seus filhos. $O$ roteiro da entrevista foi previamente elaborado pelas pesquisadoras e embasado na literatura sobre $o$ tema, contendo informações acerca da qualidade da relação fraternal e da presença ou não de responsabilidade de cuidado entre os irmãos (para mais detalhes, consultar Almeida, 2014).

\section{Procedimentos}

Após a aprovação do projeto pelo Comitê de Ética em Pesquisa com Seres Humanos da Universidade Federal de Juiz de Fora, sob o parecer no 311.444 , deu-se início ao recrutamento das famílias. O processo de seleção dos participantes foi efetuado de forma diferenciada para as famílias com filhos com SD e para aquelas com filhos com DT. No primeiro caso, selecionaram-se escolas da rede pública e privada de ensino do município que tinham alunos com SD matriculados, e, através destas, as famílias foram contatadas. Selecionadas as famílias com filhos com SD, iniciou-se o recrutamento das famílias com filhos com DT, que foi realizado através de três vias: indicação das famílias com filhos com SD; contato com escolas e creches; e indicação de terceiros. Destaca-se que, com o intuito de emparelhar as famílias da amostra caso a caso, para a seleção das famílias com filhos com DT foram levados em consideração as características das famílias com filhos com SD, especialmente no que se refere: à idade da criança ou adolescente com SD e de seu irmão; ao sexo da criança ou adolescente com SD e seu irmão; às características demográficas similares entre as famílias, tais como,

Tabela 1

Caracterização das díades de irmãos, por idade e sexo.

\begin{tabular}{lccccccc}
\hline & \multicolumn{3}{c}{ Idade (em anos) } & \multicolumn{2}{c}{ Sexo das díades } \\
\cline { 2 - 5 } Díades de irmãos & \multicolumn{3}{c}{ FSD } & \multicolumn{3}{c}{ FDT } & SD \\
\cline { 2 - 5 } & I-SD & I-DT & I-DT1 & I-DT2 & & DT \\
\hline D1 & 5 & 10 & 5 & 9 & Feminino & Feminino \\
D2 & 6 & 10 & 6 & 11 & Feminino & Feminino \\
D3 & 10 & 13 & 8 & 11 & & Masculino & Masculino \\
D4 & 13 & 9 & 15 & 11 & & Masculino & Masculino \\
\hline
\end{tabular}


número de membros e renda familiar. Todas as famílias selecionadas foram contatadas pelo telefone pela pesquisadora e auxiliares que esclareceram os objetivos da pesquisa e solicitaram o consentimento de forma oral. Aos que consentiram oralmente, foi marcada uma primeira visita à residência em dias e horários disponibilizados pelas famílias. A coleta de dados ocorreu na residência dos participantes e consistiu em duas visitas domiciliares. Na primeira visita, informações acerca da pesquisa foram esclarecidas aos genitores, obtiveram-se os consentimentos livres e esclarecidos do pai e da mãe, e a mãe respondeu ao questionário de caracterização do sistema familiar. Na segunda visita, os genitores responderam, separadamente, o roteiro da entrevista.

A análise dos resultados seguiu as especificidades de cada instrumento utilizado. Os dados coletados por meio de questionário foram tabulados, permitindo a elaboração de análises por meio de estatística descritiva. As entrevistas foram gravadas em áudio e transcritas na íntegra. A análise das entrevistas foi realizada com base na proposta de Dessen e Cerqueira-Silva (2009), que prevê a construção do Sistema Integrado de Categorias, o qual pressupõe: seleção e exploração do material, denominada pré-análise; codificação; agrupamento dos temas; formação das categorias síntese; classificação dos temas; definição das categorias; revisão do sistema preliminar e elaboração do sistema integrado (definitivo) de categorias; e validação do sistema integrado de categorias, a partir da análise de juízes. As categorias foram definidas operacionalmente $\mathrm{e}$ podem ser detalhadamente consultadas em Almeida (2014). Foi construído um dicionário de definição, procedendo à análise de juízes, tendo sido esta realizada por uma pesquisadora com experiência em análise de conteúdo e conhecimentos na área de DI e família.

\section{Resultados}

Inicialmente, são apresentadas as características das famílias participantes do estudo considerando sua composição e estrutura, bem como as atividades da rotina diária, dentre outros aspectos sobre o funcionamento e a dinâmica familiar a partir dos dados do questionário de caracterização familiar. Em seguida, são apresentados os resultados das entrevistas com os genitores, especificamente a respeito da relação fraternal. Destaca-se que, para melhor descrição dos resultados, as famílias com um membro com SD são identificadas com as letras FSD seguidas por um número, F-SD1, F-SD2 e assim por diante, enquanto as famílias com ambos os membros com DT são identificadas com as letras F-DT seguidas por um número, F-DT1, F-DT2 e assim por diante. Por ser um estudo comparativo, o número de cada família, em ambos os grupos, indica que aquelas são famílias que apresentam características similares. Portanto, F-SD1 apresenta características semelhantes à família F-DT1. Os pais são identificados com a letra $\mathrm{P}$, as mães com a letra $\mathrm{M}$, seguidas pelo código correspondente ao de sua família, assim P-SD1 é o pai em F-SD1; M-DT2 é a mãe em F-DT2, e assim por diante.

\section{Caracterização da dinâmica e do funcionamento familiar}

A rotina das famílias durante a semana era semelhante. Em geral, o pai ou ambos os genitores trabalhavam fora de casa, em período integral, só retornando à noite. Todas as crianças e adolescentes, em ambos os tipos de família, frequentavam o ensino regular em escola pública ( $\mathrm{FSD}-\mathrm{n}=2$; FDT $-\mathrm{n}=3$ ) ou privada ( $F S D-n=6$; FDT $-n=5$ ). As atividades de lazer eram similares para ambos os tipos de famílias $(n=8)$, sendo estas realizadas mais frequentemente nos finais de semana, tendo, em geral, a participação do pai, da mãe e dos filhos $(n=6)$, somente da mãe e dos filhos $(n=1)$ e uma mãe relatou que não costuma ter momentos de lazer fora do ambiente doméstico. Uma família (FDT) relatou que, além de todos os membros da família, há também a participação de amigos nas atividades de lazer. Verifica-se haver similaridade entre as famílias no tocante às idades dos membros das díades, idades dos genitores, renda familiar e rotina diária.

Em relação às atividades domésticas, as mães sozinhas são as principais responsáveis por estas funções nas famílias SD e DT, seguidas pelo pai. Além disso, nas famílias SD $(n=2)$ os filhos com e sem SD são responsáveis por "limpar a casa", enquanto que, em uma família DT, o filho mais velho tem essa responsabilidade. As atividades compartilhadas podem ser observadas na Tabela 2.

Quanto às tarefas relacionadas aos cuidados com os filhos, há variedade nos relatos. Os relatos mostram que as pessoas com SD realizam diversas atividades sozinhas $(\mathrm{n}=9)$ mais do que os participantes com DT ( $\mathrm{n}=7$ ) no grupo de comparação. As mães são as responsáveis por supervisionar/acompanhar grande parte das atividades de cuidados com o participante focal nas famílias FDT $(n=10)$, mais do que as mães nas famílias FSD ( $n=6)$, indicando que o participante com 
SD é independente em relação a algumas atividades de cuidados. Verifica-se que o pai sozinho é responsável apenas por ler histórias para o filho em uma família DT. Ambos os genitores compartilham a atividade de levar os filhos às atividades de lazer (SD $-\mathrm{n}=3$; DT $-\mathrm{n}=2)$, havendo, portanto, mais participação, nessa atividade, dos pais nas famílias SD. A Tabela 3 especifica as atividades e os membros responsáveis por supervisioná-las.

\section{Relações fraternais: a perspectiva dos genitores}

Os pais e as mães caracterizaram a relação entre seus filhos como sendo "Mista", ou seja, ora havendo conflito, ora havendo amistosidade (SD - mãe: $\mathrm{n}=1$, pai: $\mathrm{n}=2$; DT - mãe: $n=3$, pai: $n=2$ ), somente "Amistosa" (SD - mãe: $\mathrm{n}=3$, pai: $\mathrm{n}=1$; DT - mãe: $\mathrm{n}=1$, pai: $\mathrm{n}=2$ ) e "Conflituosa" (SD - pai: $\mathrm{n}=1$ ), como se verificam os relatos abaixo:
Eu acho que existe muita amizade, mas, ao mesmo tempo, que existe amizade, existe também uma disputa muito grande de atenção dos pais (P-DT3). [...] elas brincam muito, né? A [irmã com DT] conversa muito com ela [irmã com SD]. Brigam também, bastante (M-SD1).

Ah, a melhor possível. A relação de amigo, de irmão (P-SD4).

Tanto nas famílias SD quanto nas famílias DT, os genitores, frequentemente, relataram a existência da liderança na relação fraternal (SD - mãe: $\mathrm{n}=4$, pai: $\mathrm{n}=2$; DT mãe: $\mathrm{n}=4$, pai: $\mathrm{n}=2$ ). Nas famílias com filhos com SD, todos os genitores que afirmaram haver liderança na relação entre os filhos, com exceção de uma mãe, indicaram o filho com DT como o líder. Já nas famílias com filhos com DT, a maioria dos genitores

Tabela 2

Responsáveis por realizar as tarefas domésticas, segundo cada tipo de família

\begin{tabular}{|c|c|c|c|c|c|c|c|c|c|c|c|c|}
\hline \multirow{3}{*}{ Atividades dométicas } & \multicolumn{2}{|c|}{ Mãe } & \multicolumn{2}{|c|}{ Pai } & \multicolumn{2}{|c|}{ Irmão } & \multicolumn{2}{|c|}{ Irmão focal } & \multicolumn{2}{|c|}{ Empregada } & \multicolumn{2}{|c|}{ Outros } \\
\hline & SD & DT & SD & DT & $\mathrm{SD}$ & DT & $\mathrm{SD}$ & DT & $\mathrm{SD}$ & DT & SD & DT \\
\hline & $\mathrm{n}$ & $\mathrm{n}$ & $\mathrm{n}$ & $\mathrm{n}$ & $\mathrm{n}$ & $\mathrm{n}$ & $\mathrm{n}$ & $\mathrm{n}$ & $\mathrm{n}$ & $\mathrm{n}$ & $\mathrm{n}$ & $\mathrm{n}$ \\
\hline Limpar a casa & 4 & 3 & 2 & 2 & 2 & 1 & 2 & - & - & - & 2 & 1 \\
\hline Cozinhar & 4 & 3 & 2 & - & - & - & - & 1 & 1 & - & 1 & 1 \\
\hline Lavar e passar & 3 & 3 & 1 & 1 & - & - & - & - & 1 & - & 1 & 1 \\
\hline Comprar comida & 3 & 3 & 4 & 2 & - & - & - & - & - & - & - & - \\
\hline Orientar a empregada & 1 & 1 & - & - & 1 & - & - & - & - & - & - & - \\
\hline Total & 15 & 13 & 9 & 5 & 3 & 1 & 2 & 1 & 2 & - & 4 & 3 \\
\hline
\end{tabular}

Nota. "Irmão focal" corresponde ao irmão com SD e nas famílias DT é o irmão de sexo e idade correspondentes ao participante com SD.

Tabela 3

Responsáveis por acompanhar/supervisionar as tarefas desenvolvidas com o filho, segundo cada tipo de família

\begin{tabular}{|c|c|c|c|c|c|c|c|c|c|c|c|c|}
\hline \multirow{3}{*}{ Tarefas } & \multicolumn{2}{|c|}{ Irmão focal } & \multicolumn{2}{|c|}{ Mãe } & \multicolumn{2}{|c|}{ Pai } & \multicolumn{2}{|c|}{ Genitores } & \multicolumn{2}{|c|}{ Madrinha } & \multicolumn{2}{|c|}{ Irmão } \\
\hline & $\mathrm{SD}$ & DT & SD & DT & SD & DT & SD & DT & SD & DT & SD & DT \\
\hline & $\mathrm{n}$ & $\mathrm{n}$ & $\mathrm{n}$ & $\mathrm{n}$ & $\mathrm{n}$ & $\mathrm{n}$ & $\mathrm{n}$ & $\mathrm{n}$ & $\mathrm{n}$ & $\mathrm{n}$ & $\mathrm{n}$ & $\mathrm{n}$ \\
\hline Alimentação & 3 & 2 & - & 2 & - & - & - & - & 1 & - & - & - \\
\hline Banho & 2 & 2 & 1 & 2 & - & - & - & - & 1 & - & - & - \\
\hline Colocar para dormir & 2 & 1 & 1 & 1 & - & - & 1 & 2 & - & - & - & - \\
\hline Levar à escola & 1 & - & 2 & 3 & - & - & - & - & 1 & - & - & - \\
\hline Ler histórias & 1 & 1 & 1 & 1 & - & 1 & - & - & - & - & 1 & 1 \\
\hline Levar às atividades de lazer & - & 1 & 1 & 1 & - & - & 3 & 2 & - & - & - & - \\
\hline Total & 9 & 7 & 6 & 9 & - & 1 & 4 & 4 & 3 & - & 1 & 1 \\
\hline
\end{tabular}

Nota. "Irmão focal" corresponde ao irmão com SD e nas famílias FDT corresponde ao irmão de sexo e idade equivalentes ao participante com SD. 
$(n=5)$ indicou que o irmão mais velho assumia este papel, conforme exemplificam os relatos a seguir:

Geralmente, eu acho que o mais velho comanda e o mais novo tá sempre em busca de copiar o mais velho [...] (P-DT3).

Não, todas as duas têm [...] (P-DT2).

São bem iguais nas relações (P-SD3).

No que se refere à atividade de cuidado entre os irmãos, a maioria dos genitores relata que existe a responsabilidade de um filho cuidar do outro. Nas famílias SD, os genitores relataram que o filho com DT tem a responsabilidade de cuidar do irmão com SD (mãe: $\mathrm{n}=4$, pai: $\mathrm{n}=4$ ). Além disso, três mães e dois pais afirmaram que o filho com SD também tem a responsabilidade de cuidar do irmão. Já nas famílias com filhos com DT, os genitores atribuem ao filho mais velho a responsabilidade de cuidado com o irmão (mãe: $n=3$, pai: $\mathrm{n}=3$ ), sendo que destes genitores, uma mãe e um pai indicam que o filho mais novo também é responsável pelo cuidado com o irmão mais velho. Nestas famílias, uma mãe e um pai não responderam à questão. Seguem abaixo exemplos de relatos em que essas características podem ser identificadas:

Sim. A mais velha tem que cuidar da mais nova, olhar, zelar por ela (P-DT2).

Eu acho que o mais velho se sente mais responsável pelo mais novo (P-DT3).

[...] ele [irmão com DT] toma muita conta do [irmão com SD]. Ele [irmão com DT] é muito preocupado com ele [irmão com SD],... zela pelo irmão (P-SD4).

Ela [irmã com DT] procura ficar sempre perto dela [irmã com SD]. É, assim, num lugar perigoso segura na mãozinha da irmã [irmã com $\mathrm{SD}](\mathrm{M}-\mathrm{SD} 1)$.

\section{Discussão}

Não há dúvidas quanto à importância da análise de diferentes fatores na compreensão da dinâmica e funcionamento familiar. Os resultados deste estudo indicam similaridades e diferenças no funcionamento e dinâmica de famílias com e sem filhos com SD. As similaridades podem ser obser- vadas na rotina diária das famílias, no fato de a mãe ser a principal responsável pelas tarefas domésticas e atividades de cuidado com os filhos, bem como no compartilhamento da responsabilidade pelas atividades de lazer por mãe e pais em ambas as famílias. Evidenciou-se, também, que a relação fraternal foi caracterizada como positiva na maioria das famílias, o que indica que a presença de um irmão com SD não prejudica a amistosidade da relação. Além disso, observou-se que os irmãos das crianças e adolescentes com SD, independentemente de sua idade e ordem de nascimento, desempenham o papel de cuidador do irmão e líder da relação. Por outro lado, nas famílias com filhos com DT, o irmão mais velho é quem assume esse papel, o que coincide com a literatura no que se refere às diferenças na relação fraternal nessas famílias.

Verificaram-se diferenças em algumas variáveis, tais como, a natureza do trabalho dos genitores. Todos os pais trabalhavam mais que 8 horas diárias, mas aqueles do grupo FDT trabalhavam mais horas do que os do grupo FSD e, comparando o tempo de trabalho entre pais e mães, aqueles, nas famílias FDT trabalhavam o dobro das mães nesse mesmo grupo. Ressalta-se que todas as mães em FSD trabalhavam fora de casa e somente uma (FDT) não trabalhava. No entanto, as famílias FSD perfaziam uma renda familiar, em média, um pouco menor que as FDT. É fundamental destacar que o excesso de horas no trabalho tem impacto na rotina da família, uma vez que, ambos os genitores se sobrecarregam com o trabalho e, no caso das mães, a estas se acrescem as responsabilidades com os afazeres domésticos e com os cuidados com os filhos, como mostram os resultados, o que corrobora os dados da literatura (Pereira-Silva, Dessen, \& Barbosa, 2015; Pereira-Silva, Oliveira, \& Rooke, 2015; Rabelo, 2013; Rooke, 2014; Wagner, Predebon, Mosmann, \& Verza, 2005). Entretanto, a sobrecarga tem impactos importantes sobre a saúde da pessoa, bem como para o funcionamento da família (Stoneman, 2009).

Esse resultado configura-se uma similaridade entre as famílias, ou seja, em ambos os grupos as mães são as principais responsáveis pelas tarefas de casa e pelos filhos. As atividades de lazer são realizadas nos fins de semana, o que corrobora outros estudos com famílias com e sem filho com SD (Pereira-Silva, \& Dessen, 2006). Pode-se afirmar que 
em ambas as famílias o papel do pai tem sido mais de provedor, isto é, aquele que arca com a maior parte do sustento da família (Dessen, 2010), apesar de se verificar que somente uma mãe não trabalha fora de casa. Devido a essa configuração da dinâmica e funcionamento das famílias, o pai compartilha pouco com a esposa as atividades de rotina diária da casa e cuidados com filhos nessas famílias, principalmente devido ao fato de os pais terem uma carga horária diária acima de 8 horas de trabalho. Esses dados são convergentes com outros estudos (Pereira-Silva et al., 2015; Pereira-Silva et al., 2015; Rabelo, 2013; Rooke, 2014), mostrando pais participando mais das atividades de lazer com a família e compartilhando menos nas atividades domésticas. Apesar disso, Wagner et al. (2005) identificaram que as tarefas domésticas estão sendo compartilhadas, progressivamente, entre os genitores em grande parte das famílias do Brasil.

No que tange às tarefas desenvolvidas pelas pessoas com SD, ressalta-se que os relatos dos genitores mostram que estas têm realizado diversos afazeres domésticos de forma autônoma e independente mais do que os participantes nas famílias DT. Verifica-se, portanto, que as crianças e adolescentes com SD do presente estudo mostram-se bastante autônomos e independentes em atividades de vida diária. Provavelmente, a crescente autonomia das crianças e adolescentes com SD deve-se em parte ao compromisso dos genitores no que se refere ao engajamento na promoção do desenvolvimento e da saúde do filho (Rooke, 2014), bem como devido ao acesso aos programas de estimulação precoce e de outras intervenções, tendo implicações na qualidade de vida dessas pessoas (Hallal, Marques, \& Braccialli, 2008; Mattos, \& Bellani, 2010).

No geral, a relação fraternal, na perspectiva dos genitores, ora é conflituosa ora é amistosa, isto é, Mista. Vale ressaltar que este resultado indica que a relação fraternal nas famílias investigadas é positiva, já que a relação entre irmãos é tipicamente caracterizada por amistosidade, proximidade e, ao mesmo tempo, por algum nível de conflito, que, embora presente, não prejudica o vínculo saudável entre os irmãos. Pais e mães no grupo FDT apresentaram relatos mais similares quanto à sua percepção da relação fraternal dos filhos do que os genitores no outro grupo. As análises entre os grupos mostram mais genitores nas famílias SD ten- dendo a perceber essa relação como Amistosa do que como Mista. Destaca-se que um pai no grupo SD percebe a relação fraternal como Conflituosa apenas. Esses dados convergem com os da literatura acerca do fato de que a relação entre a díade de irmãos SD-DT tende a ser positiva, com características de amistosidade (Abramovitch et al., 1987; Orsmond, \& Seltzer, 2007; Pereira-Silva, 2003) mais do que conflitos. Corrobora também os dados de Cuskelly e Gunn (2003), sugerindo uma tendência das famílias SD em relatar aspectos mais saudáveis das interações fraternais. Aliado a todos esses aspectos, diferentemente de alguns resultados encontrados por Mulroy et al. (2008), os genitores com filho com SD não relataram desvantagens para o filho com DT devido à sua relação com o irmão com SD. Os resultados indicam mais vantagens. Entretanto, é importante destacar a necessidade de estudos observacionais com a díade de irmãos, obtendo assim dados das interações fraternais e não somente por meio dos relatos dos genitores.

Os genitores, em ambas as famílias, percebem haver liderança na relação fraternal e assim como apontado em alguns estudos, nas famílias SD, os irmãos com DT assumem o papel de irmão mais velho, independentemente da idade e da ordem de nascimento (Almeida, 2014; Burke, 2010; Núnez, \& Rodríguez, 2005; Pereira-Silva, 2003; Stoneman, 2005), sendo os líderes da relação. Entretanto, ressalta-se que na maioria das famílias SD da presente investigação, o irmão com DT era o primogênito da díade fraternal. Nas famílias DT, em geral, os primogênitos foram descritos como sendo os que mais lideram a relação fraternal, corroborando a literatura sobre o tema (Brody, 1998).

Quanto à responsabilidade de cuidados entre os irmãos, a maioria dos pais e mães nas famílias SD considera que os irmãos com DT são responsáveis pelo cuidado de seus irmãos com SD, conforme indica a literatura (Abramovitch et al., 1987; Brody et al., 1991; Hannah, \& Midlarsky, 2005). Vale ressaltar que quatro genitores relataram que o filho com SD também tem responsabilidade de cuidado do irmão. Esse dado não foi encontrado na literatura de irmãos e indica a necessidade de investigação de fatores culturais influenciando nos valores parentais e nos modos de criação de filhos. Além disso, pode indicar o quanto as pessoas com SD apresentam-se mais independentes e autôno- 
mas, o que faz sentido para os genitores atribuírem responsabilidades a esses filhos também e não somente àquele com DT. Nas famílias DT, os irmãos mais velhos são responsáveis pelo cuidado de seus irmãos mais novos. De acordo com Cuskelly e Gunn (2003) e McHale e Gamble (1989), nessas famílias, há uma tendência do filho primogênito cuidar dos outros, o que pode ser evidenciado também no Brasil (Dellazzana, \& Freitas, 2010; Ferreira, \& Mettel, 1999). No entanto, devido à variabilidade cultural esta tendência pode ser modificada (Cuskelly, \& Gunn, 2003). Portanto, estudos transculturais seriam interessantes para se verificar a dinâmica das relações fraternais em diferentes culturas.

Em relação à diferença de gênero no que se refere aos cuidados entre os filhos, Seltzer, Begun, Seltzer e Krauss (1991) indicam que as mães tendem a designar uma irmã como cuidadora, independentemente se a díade fraternal tiver alguma deficiência. Desta maneira, pressupõe-se que haverá diferenças de gênero nas relações entre os irmãos acerca da distribuição de tarefas. No entanto, todos os irmãos das crianças e adolescente com SD participantes, de ambos os sexos, são apontados pelos genitores como responsáveis pelo cuidado de seu/sua irmão(ã). Estes achados corroboram os resultados de Cuskelly e Gunn (2003) que também não encontraram evidências que comprovassem diferenças em relação ao gênero e ao papel desempenhado pelo irmão com DT.

Destaca-se a importância da inclusão do pai como respondente no presente estudo, bem como de se ter dados de dois tipos diferentes de famílias, proporcionando uma análise mais completa do grupo e não repetindo um erro metodológico de excluir o pai das pesquisas, como ocorria há décadas atrás (Dessen, \& Lewis, 1998). Apesar de neste estudo mães e pais, nas mesmas famílias, terem percepções semelhantes sobre muitos aspectos da relação desenvolvida entre os filhos, no estudo de Cuskelly e Gunn (2006) foram encontradas diferenças entre os relatos dos genitores.

Ressalta-se a importância de se ter uma compreensão do grupo de acordo com a perspectiva sistêmica, buscando-se investigar todos os membros da família. Assim, a não inclusão dos irmãos com e sem SD como respondentes pode ser considerada uma limitação deste estudo e deve ser uma prioridade para as próximas pesquisas. Contudo, observa-se a dificuldade em investigar crianças e adolescentes com SD por meio de instrumentos de relato oral, embora não haja dúvidas quanto à importância da participação deles nos estudos, conforme enfatizam Cerqueira-Silva (2011) e Rabelo (2013). É, pois, necessário que os pesquisadores desenvolvam instrumentos e técnicas de coleta de dados mais adequados a fim de tornar possível a participação de pessoas com SD como informantes de pesquisa.

Outra limitação deste estudo é o pequeno número de famílias participantes, o que impede uma análise mais complexa dos resultados, bem como a generalização dos dados para as demais famílias brasileiras. No entanto, esta investigação pode ser considerada um importante impulso à pesquisa comparativa com irmãos no Brasil, já que elas são raras em nosso país e difíceis de serem conduzidas em razão da quantidade de variáveis que devem ser controladas. Estudos comparativos com amostras maiores podem ser importantes para a melhor compreensão das similaridades e diferenças entre os distintos tipos de família no contexto brasileiro. Seria interessante, também, que as próximas pesquisas controlem o sexo dos irmãos a fim de averiguar se há diferenças entre meninas e meninos no que concerne ao papel desempenhado e à qualidade da interação.

Conforme observado, os resultados deste estudo indicam similaridades e diferenças no funcionamento e dinâmica de famílias com e sem filhos com SD. As similaridades podem ser observadas na rotina diária das famílias, no fato de a mãe ser a principal responsável pelas tarefas domésticas e atividades de cuidado com os filhos, bem como no compartilhamento da responsabilidade pelas atividades de lazer por mãe e pais em ambas as famílias. Percebeu-se, também, que a relação fraternal foi caracterizada como positiva na maioria das famílias, o que indica que a presença de um irmão com SD não prejudica a amistosidade da relação. Além disso, conforme esperado, observou-se que os irmãos das crianças e adolescentes com SD, independentemente de sua idade e ordem de nascimento, desempenham o papel de cuidador do irmão e líder da relação. Por outro lado, nas famílias com filhos com DT, o irmão mais velho é quem assume esse papel, o que coincide com a literatura no que se refere às diferenças na relação fraternal nessas famílias. 


\section{Referências}

Abramovitch, R., Stanhope, L., Pepler, D., \& Corter, C. (1987). The influence of Down's syndrome on sibling interaction. Journal of Child Psychology and Psychiatry and Allied Disciplines, 28(6), 865-879. https://doi.org/10.1111/j.1469-7610.1987.tb00675.x

Aguayo, C. M. (2010). Revisión teórica sobre el ajuste psicológico y emocional de los hermanos de personas con discapacidad intelectual. Revista Española sobre Discapacidad Intelectual, 41, 60-78. Recuperado de http://sid. usal.es/idocs/F8/ART16113/235-4\%20Moreno.pdf

Almeida, B. R. (2014). Interações fraternais em famílias de crianças e adolescentes com síndrome de Down (Dissertação de mestrado). Instituto de Ciências Humanas da Universidade Federal de Juiz de Fora, Juiz de Fora, MG.

Bailey Jr., D. B. (2007). Introduction: family adaptation to intellectual and developmental disabilities. Mental Retardation and Developmental Disabilities Research Reviews, 13, 291-292. https://doi.org/10.1002/mrdd.20168

Brody, G. H. (1998). Sibling relationship quality: its causes and consequences. Annual Review of Psychology, 49, 1-24. https://doi.org/10.1146/annurev.psych.49.1.1

Brody, G. H., Stoneman, Z., Davis, C. H., \& Crapps, J. M. (1991). Observations of the role relations and behavior between older children with mental retardation and their younger siblings. American Journal on Mental Retardation, 95(5), 527-536.

Burke, P. (2010). Brother and sisters of disabled children: the experience of disability by association. British Journal of Social Work, 40(6), 1681-1699. https://doi.org/10.1093/bjsw/bcp088

Casarin, S. (1999). Aspectos psicológicos na síndrome de Down. In J. S. Schwartzman (Org.), Síndrome de Down (pp. 263-285). São Paulo, SP: Mackenzie.

Cerqueira-Silva, S. (2011). Os atendimentos às famílias com necessidades especiais: Uma análise das políticas, dos programas, dos serviços e da participação dos familiares (Tese de Doutorado). Instituto de Psicologia da Universidade de Brasília, Brasília, DF.

Choi, H., \& Van Riper, M. (2014). Maternal perceptions of sibling adaptation in Korean families of children with Down syndrome. Journal of Intellectual Disability Research, 58(10), 962-977. https://doi.org/10.1111/jir.12126

Cuskelly, M. (2016). Contributors to adult sibling relationships and intention to care of siblings of individuals with Down syndrome. American Journal on Intellectual and Developmental Disabilities, 121(3), 204-218. https:// doi.org/10.1352/1944-7558-121.3.204

Cuskelly, M., \& Gunn, P. (2003). Sibling relationships of children with Down syndrome: Perspectives of mothers, fathers, and siblings. American Journal of Mental Retardation, 108(4), 234-244. https://doi.org/10.1352/0895-8017(2003)108<234:SROCWD>2.0.CO;2

Cuskelly, M., \& Gunn, P. (2006). Adjustment of children who have a sibling with Down syndrome: perspectives of mothers, fathers, and children. Journal of Intellectual Disability Research, 50(Pt 12), 917-925. https:// doi.org/10.1111/j.1365-2788.2006.00922.x

Dellazzana, L. L., \& Freitas, L. B. L. (2010). Um dia na vida de irmãos que cuidam de irmãos. Psicologia: Teoria e Pesquisa, 26(4), 595-603. https://doi.org/10.1590/S0102-37722010000400003

Dessen, M. A. (2010). Estudando a família em desenvolvimento: Desafios conceituais e teóricos. Psicologia: Ciência e Profissão, 30(n spe), 202-219. https:// doi.org/10.1590/S1414-98932010000500010

Dessen, M. A. (2009). Questionário de caracterização do sistema familiar. In L. Weber, \& M. A. Dessen (Eds.), Pesquisando a família: instrumentos para coleta e análise de dados (pp.102-114). Curitiba, PR: Juruá.

Dessen, M. A., \& Cerqueira-Silva, S. (2009). Desenvolvendo sistemas de categorias com dados de entrevistas. In L. Weber, \& M. A. Dessen (Eds.), Pesquisando a Família: Instrumentos para coleta e análise de dados (pp. 43-56). Curitiba, PR: Juruá.

Dessen, M. A., \& Lewis, C. (1998). Como estudar a família e o "pai". Paidéia, 8(14), 105-121. https://doi.org/10.1590/S0103-863X1998000100009 
Dodd, D. C. H., Zabriskie, R. B., Widmer, M. A., \& Eggett, D. (2009). Contributions of family leisure to family functioning among families that include children with developmental disabilities. Journal of Leisure Research, 41(2), 261-286. Recuperado de http://www.nrpa.org/globalassets/journals/jlr/2009/volume-41/jlr-volume-41-number-2-pp-261-286.pdf

Ferreira, E. A. P., \& Mettel, T. P. L. (1999). Interação entre irmãos em situação de cuidados formais. Psicologia: Reflexão e Crítica, 12(1), 133-146. https://doi.org/10.1590/S0102-79721999000100009

Floyd, F. J., Costigan, C. L., \& Richardson, S. S. (2016). Sibling relationships in adolescence and early adulthood with people who have intellectual disability. American Journal on Intellectual and Developmental Disabilities, 121(5), 383-397. https://doi.org/10.1352/1944-7558-121.5.383

Gomes,V.F.,\&Bosa,C. (2004).Estresseerelaçõesfamiliaresnaperspectivadeirmãosdeindivíduos comtranstornosglobaisdodesenvolvimento. EstudosdePsicologia,(3) 9,553-561.https://ddoi.org/10.1590/S1413-294X2004000300018

Grisante, P. C., \& Aiello, A. L. R. (2012). Interações familiares: observação de diferentes subsistemas em família com uma criança com síndrome de Down. Revista Brasileira de Educação Especial, 18(2), 195-212. https://doi.org/10.1590/S1413-65382012000200003

Hallal, C. Z., Marques, N. R., \& Braccialli, L. M. P. (2008). Aquisição de habilidades funcionais na área de mobilidade em crianças atendidas em um programa de estimulação precoce. Revista Brasileira de Crescimento e Desenvolvimento Humano, 18(1), 27-34. Recuperado de http://pepsic.bvsalud.org/scielo.php?script=sci_arttext\&pid=S0104-12822008000100005\&lng= pt\&nrm=iso\&tlng=pt

Hannah, M. E., \& Midlarsky, E. (2005). Helping by siblings of children with mental retardation. American Journal of Mental Retardation, 110(2), 87-99. https://doi.org/10.1352/0895-8017(2005)110<87:HBSOCW>2.0.CO;2

Henn, C. G., \& Sifuentes, M. (2012). Paternidade no contexto das necessidades especiais: Revisão sistemática da literatura. Paidéia, 22(51), 131-139. https://doi.org/10.1590/S0103-863X2012000100015

Inam, A., \&Zehra, A. (2012). Effect of mentally retarded children on their non retarded female siblings (12-18 years). International Journal of Social Science, 1, 1-19. https://doi.org/10.7718/ijss.vlil.1

Iriarte, C., \& Ibarrola-García, S. (2010). Revisión de estudios sobre la vivencia emocional de la discapacidad intelectual por parte de los hermanos. Estudios sobre Educación, 19, 53-75. Recuperado de https://www.unav.edu/ publicaciones/revistas/index.php/estudios-sobre-educacion/article/view/4580/3948

Lemes, L. C., \& Barbosa, M. A. M. (2007). Comunicando à mãe o nascimento do filho com deficiência. Acta Paulista de Enfermagem, 20(4), 441-445. Recuperado de http://www2.unifesp.br/acta/pdf/v20/n4/v20n4a9.pdf

McHale, S. M., \& Gamble, W. C. (1989). Sibling relationships of children with disabled and nondisabled brothers and sisters. Developmental Psychology, 25(3), 421-429. https://doi.org/10.1037/0012-1649.25.3.421

Mandleco, B., \& Webb, A. E. M. (2015). Sibling perceptions of living with a young person with Down syndrome or autism spectrum disorder: an integrated review. Journal for Specialists in Pediatric Nursing, 20(3), 138-156. https://doi.org/10.1111/jspn.12117

Mattos, B. M., \& Bellani, C. D. F. (2010). A importância da estimulação precoce em bebês portadores de síndrome de Down: revisão de literatura. Revista Brasileira de Terapias e Saúde, 1(1), 51-63. https://doi.org/10.7436/rbts-2010.01.01.05

Messa, A. A., \& Fiamenghi Jr., G. A. (2010). O impacto da deficiência nos irmãos: histórias de vida. Ciência e Saúde Coletiva, 15(2), 529-538. https://doi.org/10.1590/S1413-81232010000200029

Meyers, C., \& Vipond, J. (2005). Play and social interactions between children with developmental disabilities and their siblings: a systematic literature review. Physical \& Occupational Therapy in Pediatrics, 25(1-2), 81-103. https://doi.org/10.1080/J006v25n01_06

Mulroy, S., Robertson, L., Aiberti, K., Leonard, H., \& Bower, C. (2008). The impact of having a sibling with an intellectual disability: parental perspectives in two disorders. Journal of Intellectual Disability Research, 52(3), 216-229. https://doi.org/10.1111/j.1365-2788.2007.01005.x

Nunes, C. C. (2006). Interação entre irmãos de indivíduos com deficiência mental: o papel da idade e do apoio social da família (Dissertação de mestrado). Centro de Educação e Ciências Humanas da Universidade de São Carlos, São Carlos, SP. 
Nunes, M. D. R., \& Dupas, G. (2011). Independência da criança com síndrome de Down: a experiência da família. Revista Latino-Americana de Enfermagem, 19(4), 1-9. Recuperado de http://www.scielo.br/pdf/rlae/ v19n4/pt_18

Núnez, B., \& Rodríguez, L. (2005). Los hermanos con discapacidad: una asignatura pendiente. Buenos Aires: Associación AMAR.

Orsmond, G. I., \& Seltzer, M. M. (2007). Siblings of individuals with autism or Down syndrome: effects on adults lives. Journal of Intellectual Disability Research, 51(9), 682-696. https://doi.org/10.1111/j.1365-2788.2007.00954.x

Pereira-Silva, N. L. (2003). Famílias de crianças com e sem síndrome de Down: Um estudo comparativo das relações familiares (Tese de doutorado). Instituto de Psicologia da Universidade de Brasília, Brasília, DF.

Pereira-Silva, N. L., \& Dessen, M. A. (2006). Famílias de crianças com síndrome de Down: sentimentos, modos de vida e estresse parental. Interação em Psicologia, 10(2), 183-194. https://doi.org/10.5380/psi.v10i2.7675

Pereira-Silva, N. L., Dessen, M. A., \& Barbosa, A. J. G. (2015). Ajustamento conjugal: comparação entre casais com e sem filhos com deficiência intelectual. PsicoUSF, 20(2), 297-308. httpd://doi.org/10.1590/1413-82712015200210.

Pereira-Silva, N. L., Oliveira, L., \& Rooke, M. I. (2015). Famílias com adolescente com síndrome de Down: apoio social e recursos familiares. Avances en Psicología Latinoamericana, 32(2), 269-283. https:// doi.org/10.12804/apl33.02.2015.07

Petean, E. B. L., \& Suguihura, A. L. M. (2005). Ter um irmão especial: convivendo com a síndrome de Down. Revista Brasileira de Educação Especial, 11(3), 445-460. https://doi.org/10.1590/S1413-65382005000300009

Povee, K., Roberts, L., Bourke, J., \& Leonard, H. (2012). Family functioning in families with a child with Down syndrome: a mixed methods approach. Journal of Intellectual Disability Research, 56(10), 961-973. https:// doi.org/10.1111/j.1365-2788.2012.01561.x

Rabelo, V. C. S. (2013). O envolvimento da família e a percepção dos professores sobre a inclusão escolar de alunos com sindrome de Down (Dissertação de mestrado). Instituto de Ciências Humanas da Universidade Federal de Juiz de Fora, Juiz de Fora, MG.

Rooke, M. I. (2014). Famílias com filho com síndrome de Down: Investigando a resiliência familiar (Dissertação de mestrado). Instituto de Ciências Humanas da Universidade Federal de Juiz de Fora, Juiz de Fora, MG.

Rossetti, Z., \& Hall, S. (2015). Adult sibling relationships with brothers and sisters with severe disabilities. Research and Practice for Persons with Severe Disabilities, 40(2), 120-137. https://doi.org/10.1177/1540796915592829

Seltzer, G. B., Begun, A., Seltzer, M. M., \& Krauss, M. W. (1991). Adults with mental retardation and their aging mothers: impacts of siblings. Family Relations, 40(3), 310-317. https://doi.org/ 10.2307/585017

Shivers, C. M., \& Dykens, E. M. (2017). Adolescent siblings of individuals with and without intellectual and developmental disabilities: self-reported empathy and feelings about their brothers and sisters. American Journal on Intellectual and Developmental Disabilities, 122(1), 62-77. https://doi.org/10.1352/1944-7558-122.1.62

Soares, M. P. G., Franco, A. L. S., \& Carvalho, A. M. A. (2009). Crianças que cuidam de irmãos com necessidades especiais. Psicologia: Teoria e Pesquisa, 25, 45-54. https:// doi.org/10.1590/S0102-37722009000100006

Stoneman, Z. (2005). Siblings of children with disabilities: research themes. Mental Retardation, 43(5), 339-350. https:// doi.org/10.1352/0047-6765(2005)43[339:SOCWDR]2.0.CO;2

Stoneman, Z. (2009). Siblings of children with intellectual disabilities: Normal, average, or not too different? In L. M. Glidden \& M. M. Seltzer (Eds.), International review of research in mental retardation: families (pp. 251-296). San Diego, CA: Academic Press.

Stoneman, Z., Brody, G. H., Davis, C. H., Crapps, J. M., \& Malone, D. M. (1991). Ascribed role relations between children with mental retardation and their young siblings. American Journal on Mental Retardation, 95, 537-550.

Tomeny, T. S., Ellis, B. M., Rankin, J. A., \& Barry, T. D. (2017). Sibling relationship quality and psychosocial outcomes among adult siblings of individuals with autism spectrum disorder and individuals with intellectual disability without autism. Research in Developmental Disabilities, 62, 104-114. https://doi.org/10.1016/j.ridd.2017.01.008

Voivodic, M. A., \& Storer, M. R. S. (2002). O desenvolvimento cognitivo das crianças com síndrome de Down à luz das relações familiares. Psicologia: Teoria e Prática, 4(2), 31-40. Recuperado de http://editorarevistas.mackenzie. br/index.php/ptp/article/view/1057/773 
Psicologia: Ciência e Profissão Out/Dez. 2017 v. 37 n4, 1037-1050.

Wagner, A., Predebon,J., Mosmann, C., \&Verza,F. (2005).Compartilhar tarefas? Papéis efunções de pai emãenafamília contemporânea. Psicologia: Teoria e Pesquisa, 21(2), 181-186. https://doi.org/10.1590/S0102-3772200500020000

Nara Liana Pereira-Silva

Doutora em Psicologia - Universidade Federal de Juiz de Fora. E-mail: naraliana.silva@uff.edu.br

Sarah de Rezende Crolman

Mestranda em Psicologia - Universidade Federal de Juiz de Fora. E-mail: sarahcrolman@hotmail.com

Bruna Rocha de Almeida

Doutoranda em Psicologia - Universidade Federal de Juiz de Fora. E-mail: bruna.r.almeida@gmail.com

Mayse Itagiba Rooke

Doutoranda em Psicologia - Universidade Federal de Juiz de Fora. E-mail: mirpsi@gmail.com

Endereço para envio de correspondência:

Instituto de Ciências Humanas - Departamento de Psicologia - Rua José Lourenço Kelmer, s/nº, Campus Universitário, Bairro São Pedro. CEP: 36036-900.

Juiz de Fora - MG, Brasil.

Recebido 08/02/2016

Reformulação 13/04/2017

Aprovado 08/06/2017

Received 02/08/2016

Reformulated 04/13/2017

Approved 06/08/2017

Recebido 08/02/2016

Reformulado 13/04/2017

Aceptado 08/06/2017

Como citar: Pereira-Silva, N. L., Crolman, S. R., Almeida, B. R., \& Rooke, M. I. (2017). Relação fraternal e síndrome de Down:umestudocomparativo.Psicologia:CiênciaeProfissão,37(3),1037-1050.https://doi.com/10.1590/1982-3703000462016

How to cite: Pereira-Silva, N. L., Crolman, S. R., Almeida, B. R., \& Rooke, M. I. (2017). Sibling relationship and Down syndrome:acomparativestudy.Psicologia:CiênciaeProfissão,37(3),1037-1050.https://doi.com/10.1590/1982-3703000462016

Cómo citar: Pereira-Silva, N. L., Crolman, S. R., Almeida, B. R., \& Rooke, M. I. (2017). Relación fraternal y síndrome de Down:unestudiocomparativo.Psicologia:CiênciaeProfissão,37(3),1037-1050.https://doi.com/10.1590/1982-3703000462016 